\title{
Zika Virus and Pregnancy: A Review of the Literature and Clinical Considerations
}

\author{
Caroline Marrs, MD $\quad$ Gayle Olson, MD ${ }^{1}$ George Saade, MD ${ }^{1}$ Gary Hankins, MD ${ }^{1}$ Tony Wen, MD \\ Janak Patel, $\mathrm{MD}^{2}$ Scott Weaver, $\mathrm{PhD}^{3}$
}

${ }^{1}$ Division of Maternal-Fetal Medicine, Department of Obstetrics and Gynecology, The University of Texas Medical Branch, Galveston, Texas

2 Division of Pediatric Infectious Diseases, Department of Pediatrics, The University of Texas Medical Branch, Galveston, Texas

${ }^{3}$ Departments of Pathology and Microbiology and Immunology, The University of Texas Medical Branch, Galveston, Texas
Address for correspondence Caroline Marrs, MD, Division of MaternalFetal Medicine, Department of Obstetrics and Gynecology, The University of Texas Medical Branch, 301 University Blvd., JSA 3.400, Galveston, TX 77555 (e-mail: carolinemarrs@gmail.com).

\begin{abstract}
Keywords

- Zika virus

- pregnancy

- fetus

- transmission

- microcephaly

The latest Zika virus (ZIKV) outbreak has reached epidemic proportions as it spreads throughout South and Central America. In November 2015, the Brazilian Ministry of Health reported a 20 -fold increase in the number of cases of neonatal microcephaly, which corresponds geographically and temporally to the ZIKV outbreak. Case reports have provided some evidence of a causal link between maternal ZIKV infection, fetal microcephaly, and intracranial calcifications. The sparse data regarding ZIKV in pregnancy come solely from case reports and personal communications, and recommendations for management of ZIKV exposure during pregnancy are rapidly evolving. Our objective is to review and synthesize the current literature regarding ZIKV as it pertains to pregnancy and provide some assistance to clinicians who may have to manage a pregnant patient with potential exposure to ZIKV. We will also explore certain aspects of related viruses in pregnancy in hopes to shed light on this little-known topic.
\end{abstract}

The Brazilian Ministry of Health released a report in November 2015 announcing a sharp increase in the rate of neonatal microcephaly, a roughly 20 -fold increase from the rate in 2010-2014. ${ }^{1,2}$ A causal association with Zika virus (ZIKV) infection was proposed based on the following: (1) increased incidence rate of microcephaly coinciding with the ZIKV outbreak and (2) two reported cases of pregnant women with symptoms consistent with ZIKV infection, fetal microcephaly, and amniotic fluid positive for ZIKV by reverse transcriptase polymerase chain reaction (RT-PCR) methods. ${ }^{1}$ Subsequently, in Brazil a newborn with multiple anomalies and microcephaly died shortly after birth, and the ZIKV genome was detected in his blood and tissue samples. ${ }^{2}$ This strengthened the relationship between ZIKV infection and microcephaly. The temporal association between ZIKV and adverse outcomes has not been isolated to Brazil. In Novem-

received

February 19, 2016 accepted after revision

February 25, 2016

published online

March 7, 2016 ber 2015, in French Polynesia, health authorities reported an unusual increase in central nervous system malformations in fetuses and newborns registered in 2014-2015, the same timeframe as a ZIKV epidemic there. Although none of the pregnant mothers reported symptoms consistent with ZIKV infection, four of the mothers who were tested had positive immunoglobulin $G(\operatorname{IgG})$ serology assays for Flavivirus, a genus including ZIKV, suggesting a possible connection between the infection and the fetal brain insults. ${ }^{3}$ Speculation of vertical transmission of ZIKV during pregnancy and a causal association with birth defects resulted in a deluge of alerts, practice advisories, and memoranda from the Centers for Disease Control and Prevention (CDC), the European Centre for Disease Prevention and Control (ECDC), the American Congress of Obstetrics and Gynecology (ACOG), and the Society of Maternal Fetal Medicine (SMFM) regarding the
Copyright $\odot 2016$ by Thieme Medical Publishers, Inc., 333 Seventh Avenue, New York, NY 10001, USA. Tel: +1(212) 584-4662.
License terms 10.1055/s-0036-1580089. ISSN 0735-1631.

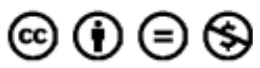


ZIKV epidemic in the Americas and its implications for

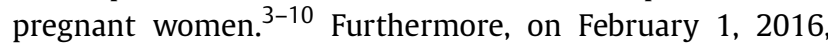
the World Health Organization (WHO) declared the ZIKV epidemic a Public Health Emergency of International Concern, ${ }^{\mathrm{i}}$ and the CDC Emergency Operations Center elevated their response to ZIKV to level 1, the highest level of activation, for only the fourth time in the history of the organization. ${ }^{11,12}$

Infection from ZIKV has reached epidemic proportions. Although there is strong supporting evidence that ZIKV infection during pregnancy is associated with adverse fetal outcomes, the magnitude of the association as well as the mechanisms and overall effects is not well understood. Our objective is to review and synthesize the current literature regarding ZIKV in pregnancy and provide some assistance to clinicians who may have to manage a patient with potential exposure to ZIKV. We will also explore certain aspects of related viruses in pregnancy to shed light on this little-known topic.

\section{Zika Virus}

ZIKV is a single-stranded RNA virus and a member of the genus Flavivirus of the family Flaviviridae, which are a type of arbovirus, or arthropod-borne viruses, believed to be transmitted by the Aedes mosquito to humans and other primates. Other Flaviviruses with a mosquito vector include dengue, Japanese encephalitis, West Nile, and yellow fever viruses. Typically, ZIKV infection is asymptomatic (80\%) or causes a relatively benign illness characterized by fever, headache, malaise, arthralgia, maculopapular rash, and conjunctivitis, similar to dengue. ${ }^{13-16}$

ZIKV was discovered in Uganda, Africa, in 1947, and shortly thereafter was associated with human disease, albeit infrequently. ${ }^{17}$ Recently, the number of cases reported from Southeast Asia and Oceania has steadily increased. ${ }^{13}$ Outbreaks were reported first in Yap Island, Micronesia, in 2007, ${ }^{16}$ and then in French Polynesia, New Caledonia, the Cook Islands, and Easter Island in 2013 and $2014,{ }^{16,18}$ consistent with a spread from Africa eastward across the Pacific. Brazil and South America represent the most recently affected areas. The ZIKV genome was first sequenced in January 2007. ${ }^{19}$ There are three subclades of ZIKV, reflecting its geographic origins. The Senegal and Uganda ZIKV represent the west and east African lineage. The 2007 ZIKV (Yap state) and the Americas ZIKV may represent the continued spread throughout Southeast Asia and the Pacific, the Asian lineage. ${ }^{20-22}$ There have been reports of rare neurologic sequelae following infection in Southeast Asia, specifically Guillain-Barré syndrome. 2,23

\footnotetext{
A Public Health Emergency of International Concern is defined as (1) constituting a health risk to other countries through international spread, (2) potentially requiring a coordinated response because it is unexpected, serious, or unusual, and (3) having implications beyond the affected country that could require immediate action. ${ }^{12}$
}

There is some concern that the current outbreak may also lead to an increase in neurologic sequelae.

While a mosquito bite is believed to be the principal form of transmission, there have been reports of less common modes, such as blood transfusion, ${ }^{24,25}$ perinatal, ${ }^{26}$ and sexual contact. $^{27,28}$ There is no gold standard for diagnosis, but RTPCR has been described as effective during the early stages of acute disease in multiple studies, with detection of the viral genome in serum, urine, saliva, and semen. ${ }^{27-29}$ RT-PCR testing is thought to remain positive in serum 4 to 7 days after symptom onset. Serologic testing is an option after the acute phase: ZIKV IgM is detectable in the serum by at least 4 days after symptom onset, but may not become positive until 2 weeks. It is unclear how long the ZIKV IgM persists in serum, but experience with other Flaviviruses would suggest 6 months. ${ }^{30}$ Serologic testing, however, has significant limitations as there is considerable cross-reactivity between ZIKV and other viruses, such as dengue and chikungunya, which are endemic in a geographic distribution similar to ZIKV. Confirmatory neutralizing antibody testing is required if the IgM testing is positive to determine likely recent ZIKV infection as opposed to prior exposure or vaccination to other viruses. There is no specific antiviral treatment or vaccination for ZIKV. Prevention of mosquito bites is the principal form of prevention, while abstinence and barrier protection have been recommended to prevent sexual transmission, especially during pregnancy. ${ }^{9}$ Until all the possible modes of transmission are better understood, universal precautions are warranted. Blood donation is of particular concern in endemic areas because roughly $80 \%$ of people with ZIKV infection are asymptomatic. During the outbreak in French Polynesia in 2013-2014, RT-PCR for ZIKV was performed in 1,505 blood donors, with 42 testing positive (3\%). Of those, 11 reported ZIKV-like symptoms 3 to 10 days after donating. ${ }^{25}$

The ECDC has issued several rapid risk assessments regarding ZIKV outbreaks in the last 2 years, in regions including French Polynesia, Brazil, and the Pacific region. ${ }^{3-5}$ The reported autochthonous (local mosquito-to-human) transmission has expanded quickly to 31 countries or territories in the world. ${ }^{5}$ At the time of submission of this article, the CDC has issued travel alerts (level 2-practice enhanced precautions) for the following regions: Cape Verde, Caribbean (Barbados, Curacao, Dominican Republic, Guadeloupe, Haiti, Jamaica, Puerto Rico, Martinique, Saint Martin, and the U.S. Virgin Islands), Central America (Costa Rica, El Salvador, Guatemala, Honduras, Nicaragua, and Panama), Mexico, the Pacific Islands (American Samoa, Samoa, Tonga), and South America (Bolivia, Brazil, Colombia, Ecuador, French Guiana, Guyana, Paraguay, Suriname, and Venezuela) (http://www.cdc.gov/zika/pregnancy/travel-health-notices. html). As of submission of this article, in the United States, local, mosquito-to-human transmission has not been reported. However, Aedes aegypti and A. albopictus mosquitos do populate large areas of the southeastern and eastern United States, respectively (map available at http://www. cdc.gov/chikungunya/resources/vector-control.html). ${ }^{31}$ There is, therefore, the potential for local trans-mission in these regions. 


\section{Zika Virus in Pregnancy: Review of the Literature}

The sparse data regarding ZIKV specifically in pregnancy come from case reports or personal communications. The exact incidence, perinatal transmission rate, and complication rate are unknown. At this time, the available evidence likely does not reflect the entire clinical and epidemiological picture given that ascertainment of cases and outcomes is incomplete. The following is a summary of the relevant literature.

Besnard et al, in 2014, described the clinical and laboratory features of two mothers and their newborns who had ZIKV infections during an outbreak in French Polynesia, which started in October 2013. ${ }^{26}$ Both mothers were symptomatic peripartum, but only one newborn showed clinical signs of infection. Maternal and neonatal serum and saliva, as well as maternal breast milk, were tested by RT-PCR. Both mothers and both neonates tested positive for ZIKV in at least one of their serum samples, which suggests both women were viremic or incubating at time of delivery.

The authors explored possible routes of the apparent perinatal transmission: transplacental, during delivery, during breastfeeding, or by close contact between mother and newborn. Their data did not support transplacental transmission. Only one infant had serum drawn on day of life 1 : this sample did not test positive for ZIKV but a sample on day 4 tested positive, suggesting transmission after birth. Large loads of ZIKV RNA were found in breast milk samples from both mothers, but no infectious virus was detected on cell culture. ${ }^{\text {ii }}$ While not definitive evidence, these data point to the possibility of breast milk transmission. The authors did not comment regarding the possibility of delivery contamination as route of transmission (and there have been no reports in the literature of testing vaginal secretions for ZIKV to date). Finally, they concluded that close contact between mother and newborn as a mode of transmission could not be excluded because both mother 1 and newborn 1 had saliva samples positive for ZIKV (however, it is not known whether the ZIKV in human saliva is infectious). The authors noted that during this large outbreak (11\% of the population with infection), they did not observe an increase in number of fetal deaths or premature births.

As part of the investigation in Brazil, Oliveira et al reported their findings in two pregnant women from Paraiba, Brazil, a state affected by the ZIKV epidemic. ${ }^{32}$ The fetuses were identified with microcephaly by prenatal ultrasound, both women reported signs and symptoms characteristic of ZIKV infection earlier in pregnancy, and although both women had

\footnotetext{
ii RT-PCR detects fragments of viral genome, while cell culture can detect infectious viral particles. Therefore, testing a sample (e.g., serum, semen, urine, saliva, or breast milk) by RT-PCR can indicate whether the individual has been or is currently infected with ZIKV, but it cannot determine whether that particular bodily fluid is infectious. This is a limitation of many of the studies reporting various body fluids testing positive by RT-PCR. RT-PCR does not inform us whether the virus is transmissible by that fluid, such as semen or breast milk.
}

negative blood testing for ZIKV, amniocentesis samples were positive for viral RNA by RT-PCR. These were the first reported evidence consistent with intrauterine transmission. Ultrasound images were included, and detailed findings were described for both fetuses: the first case demonstrated brain atrophy with coarse calcifications involving the white matter of the frontal lobes, corpus callosal and vermian dysgenesis, and enlarged cisterna magna; the second case demonstrated asymmetric hemispheres with severe unilateral ventriculomegaly, displacement of the midline, thinning of the parenchyma on the dilated side, failure to visualize the corpus callosum and thalami, thinning of the pons and brainstem, subtle calcifications around the lateral and fourth ventricles, and asymmetric eyes with cataracts and intraocular calcifications. At the time of publication by Oliveira et al, a definite association between ZIKV infection and the fetal pathology, let alone causality, was still questionable, leading the authors to conclude that other etiologies could not be excluded. The same group published additional findings from the same two cases on February 17, 2016. ${ }^{33}$ They used quantitative RT-PCR to determine the amount of ZIKV in the amniotic fluid, as well as maternal serum and urine samples. Because the amniotic fluid sample were taken weeks after the maternal illness and the maternal serum and urine samples were negative for genomic material, the authors concluded that the high intrauterine viral load resulted from persistent replication in the fetus and amniotic fluid. The complete ZIKV genome from patient 1 was sequenced, and it shared 97 to $100 \%$ genomic identity with lineages isolated during the French Polynesian outbreak in 2013. Lastly, they assessed the possibility of recombination events between ZIKV and other Flaviviruses (this has been hypothesized as an explanation for the virulence and extent of this latest ZIKV outbreak) and determined that the Brazilian ZIKV genome is not a recombinant strain. This report also provided neonatal data on the two cases. The only clinical information reported for neonate 1 was a gestational age at birth of 40 weeks and a head circumference (HC) of $30 \mathrm{~cm}$, which corresponds to almost 3 standard deviations (SD) below the mean for gestational age ( - Table 1 ). Neonate 2 was born at unstated gestational age with severe ventriculomegaly, microphthalmia, cataract, and severe arthrogryposis of the legs and arms. ${ }^{33}$

Tetro reviewed two mouse model studies from $1952^{17}$ and $1972^{34}$ that showed affinity of ZIKV to murine neuronal cells, suggesting a pathophysiological mechanism linking ZIKV infection with microcephaly. ${ }^{35}$

Subsequently, in a correspondence to the Lancet in January 2016, Ventura et al reported unilateral macular lesions in three infants with microcephaly born after the ZIKV outbreak in Brazil. No testing for ZIKV infection was performed, but the authors state that the cases fulfilled the Ministry of Health's criteria for ZIKV vertical infection (toxoplasmosis, rubella, cytomegalovirus, herpes simplex, syphilis, and HIV were all ruled out in mothers and infants). ${ }^{36}$ Meanwhile, de Paula Freitas et al published their ophthalmologic findings in a case series of 29 microcephalic infants with presumed maternal ZIKV infection in Brazil. The ages of the infants ranged from 1 to 6 months and were recruited from December 1 to 
Table 1 Mean and standard deviations (SDs) of head circumference by gestational age

\begin{tabular}{|c|c|c|c|c|c|c|}
\hline \multirow[b]{2}{*}{ Week } & \multirow[b]{2}{*}{ Mean } & \multicolumn{5}{|c|}{ Head circumference $(\mathrm{mm})$ : SD below mean } \\
\hline & & -1 & -2 & -3 & -4 & -5 \\
\hline \multicolumn{7}{|c|}{ Kurmanavicius et al } \\
\hline 12 & 72.1 & 64.5 & 56.9 & 49.3 & 41.7 & 34.1 \\
\hline 13 & 86.1 & 78.3 & 70.5 & 62.7 & 54.9 & 47.1 \\
\hline 14 & 99.9 & 91.9 & 93.9 & 75.9 & 67.9 & 59.9 \\
\hline 15 & 113.5 & 105.3 & 97.1 & 88.9 & 80.7 & 72.5 \\
\hline 16 & 126.8 & 118.3 & 109.8 & 101.3 & 92.8 & 84.3 \\
\hline 17 & 139.9 & 131.2 & 122.5 & 113.8 & 105.1 & 96.4 \\
\hline 18 & 152.7 & 143.8 & 134.9 & 126.0 & 117.1 & 108.2 \\
\hline 19 & 165.2 & 156.1 & 147 & 137.9 & 128.8 & 119.7 \\
\hline \multicolumn{7}{|c|}{ Chervenak et al } \\
\hline 20 & 175 & 160 & 145 & 131 & 116 & 101 \\
\hline 21 & 187 & 172 & 157 & 143 & 128 & 113 \\
\hline 22 & 198 & 184 & 169 & 154 & 140 & 125 \\
\hline 23 & 210 & 195 & 180 & 166 & 151 & 136 \\
\hline 24 & 221 & 206 & 191 & 177 & 162 & 147 \\
\hline 25 & 232 & 217 & 202 & 188 & 173 & 158 \\
\hline 26 & 242 & 227 & 213 & 198 & 183 & 169 \\
\hline 27 & 252 & 238 & 223 & 208 & 194 & 179 \\
\hline 28 & 262 & 247 & 233 & 218 & 203 & 189 \\
\hline 29 & 271 & 257 & 242 & 227 & 213 & 198 \\
\hline 30 & 281 & 266 & 251 & 236 & 222 & 207 \\
\hline 31 & 289 & 274 & 260 & 245 & 230 & 216 \\
\hline 32 & 297 & 283 & 268 & 253 & 239 & 224 \\
\hline 33 & 305 & 290 & 276 & 261 & 246 & 232 \\
\hline 34 & 312 & 297 & 283 & 268 & 253 & 239 \\
\hline 35 & 319 & 304 & 289 & 275 & 260 & 245 \\
\hline 36 & 325 & 310 & 295 & 281 & 266 & 251 \\
\hline 37 & 330 & 316 & 301 & 286 & 272 & 257 \\
\hline 38 & 335 & 320 & 306 & 291 & 276 & 262 \\
\hline 39 & 339 & 325 & 310 & 295 & 281 & 266 \\
\hline 40 & 343 & 328 & 314 & 299 & 284 & 270 \\
\hline 41 & 346 & 331 & 316 & 302 & 287 & 272 \\
\hline 42 & 348 & 333 & 319 & 304 & 289 & 275 \\
\hline
\end{tabular}

Source: Adapted from Kurmanavicius et al ${ }^{54}$ and Chervenak et al. ${ }^{52}$

December 21, 2015. ${ }^{37}$ Thirty-four percent of the infants had ocular abnormalities. As with the Ventura et al report, no ZIKV testing was available, but $79 \%$ of the mothers retrospectively reported symptoms consistent with ZIKV infection in their pregnancy, and testing for other congenital viruses were negative.

On February 10, 2016, the New England Journal of Medicine published a case report from Slovenia that significantly strengthens the causal link between ZIKV infection and fetal brain injury. ${ }^{38}$ The authors described the case of an expectant mother who had a febrile illness with rash at the end of the first trimester of pregnancy while living in Brazil. Ultrasound performed at 14 and 20 weeks revealed normal growth and anatomy. A 32-week ultrasound revealed growth restriction (3rd percentile), microcephaly (less than the 2 nd percentile), and intracranial as well as placental calcifications. The mother requested a termination of pregnancy and a thorough fetal autopsy was performed. Micrencephaly was confirmed with almost complete agyria, hydrocephalus, and multifocal calcifications, in the absence of any other external anatomic 
anomalies. Birth weight was at the 5 th percentile and $\mathrm{HC}$ at the 1st percentile. The fetal-placental weight ratio was less than the 3rd percentile, and there were calcifications in the placenta as well. Evaluation of fetal brain tissue resulted in finding a complete ZIKV genome sequence by RT-PCR and particles consistent with ZIKV by electron microscopy. The ZIKV genome was not found in any other autopsy tissues sampled, suggesting that the virus has a propensity for neural tissue. The authors did not report any serologic testing on the mother.

Additionally, on February 10, 2016, the CDC Morbidity and Mortality Weekly Report published a report describing further evidence of a link between ZIKV infection and microcephaly and fetal demise. ${ }^{39}$ Viral RNA and antigens were detected postmortem in brain tissues from two infants with microcephaly and in placental tissues from two early miscarriages. Histopathologic testing also indicated the presence of ZIKV in fetal tissue.

According to the preliminary analysis of the investigation conducted by the Brazil health authorities, the greatest risk of microcephaly or congenital anomalies in newborns is associated with ZIKV infection in the first trimester of pregnancy, a time of organogenesis. ${ }^{40,41}$ This is consistent with other congenital infections with neurological sequelae, such as rubella and cytomegalovirus, whose severity of disease is inversely related to gestational age at time of fetal infection. ${ }^{42,43}$

There is no evidence to suggest that ZIKV infection during pregnancy is associated with a more severe illness for the mother, has long-term effects on fertility, or is associated with adverse fetal outcomes in future pregnancies. However, longterm studies are lacking.

The CDC released additional information on February 26, 2016 , regarding cases of Zika infection in pregnancy that were detected and managed in the United States. ${ }^{44}$ Between August 1, 2015 and February 10, 2016, the CDC received 257 requests for Zika virus testing for pregnant women. Information indicating that the woman had a clinical illness consistent with Zika virus disease was provided for 151 (59\%) of the requests. Documentation for the remaining requests either did not support an illness compatible with Zika virus disease or may have been incomplete. In nine pregnant women, testing confirmed Zika virus disease, all of whom survived and did not require hospitalization. All women reported a rash, and all but one woman had at least two symptoms. Of the six pregnant women who reported symptoms during the first trimester, one pregnancy is continuing, two had early pregnancy losses, two had elective pregnancy terminations, and one delivered a live-born infant with microcephaly. The other three women had symptoms in the second or third trimester, with two delivering healthy infant, and one whose pregnancy is continuing. Zika virus RNA was detected in the specimens from both cases with early pregnancy loss. One of the pregnancy terminations had evidence of brain atrophy on fetal ultrasound and MRI, as well as Zika virus RNA on amniocentesis. As of February 24, 2016, no confirmed cases were identified among 162 pregnant women without reported symptoms. CDC has developed a registry to collect information on U.S. pregnant women with confirmed Zika virus infection and their infants. Health care providers are encouraged to discuss participation in the U.S. registry with pregnant women with Zika virus infection.

\section{Sexual Transmission of Zika Virus}

The concern for sexual transmission is based on three cases so far. The first report was of probable sexual transmission from a man to a woman a few days prior to the man's onset of symptoms. ${ }^{27}$ The second report is of infectious ZIKV isolated from semen at least 2 weeks and possibly up to 10 weeks after the onset of symptoms. Interestingly, the blood that was drawn at the same time as the semen was collected tested negative for ZIKV by RT-PCR (serologic testing was not reported), suggesting that transmission via semen is possible even with negative blood testing. ${ }^{28}$ The third report is a case of sexual transmission that occurred in Dallas, Texas, where a man who had just returned from an endemic area had intercourse with his wife, who subsequently tested positive for ZIKV. This case is currently under investigation, and hopefully longitudinal sampling will provide information on the persistence of infectious ZIKV in semen to assist with risk assessments (unpublished data).

\section{Risk of Zika Virus Transmission by Blood Transfusion}

Because pregnant and peripartum women are at risk of needing a blood transfusion, it is important to understand the potential risks during an epidemic involving pathogens that may be transmitted through blood transfusion. The concern about potential transmission of ZIKV by blood transfusion is magnified by the facts that $80 \%$ of infections are asymptomatic and that there are no licensed blood donor screening tests for ZIKV in the United States. ${ }^{45}$ There have been no confirmed cases of ZIKV infection by transfusion, but there is one credible case undergoing investigation in Brazil. ${ }^{45}$

AABB (formerly known as the American Association of Blood Banks), the professional organization involved in setting regulations for blood products, has published a bulletin on February 1, 2016, in response to the ongoing outbreaks of ZIKV, dengue virus (DENV), and chikungunya virus (CHIKV) in Mexico, the Caribbean, and Central and South America. ${ }^{45}$ The bulletin cited the finding that $3 \%$ of asymptomatic blood donors tested positive for ZIKV by RT-PCR during the 2013-2014 outbreak in French Polynesia to highlight the plausibility of transmission through blood transfusion. ${ }^{25}$ To determine the burden on the United States' blood supply, it cited data from two nationwide surveys that show that as many as $2.25 \%$ of otherwise qualified blood donors in the United States and Canada had traveled to the western hemisphere in the 28 days before their donation. ${ }^{46}$ The AABB recommended that self-deferral from donation until 28 days after travel to Mexico, the Caribbean, or Central or South America should be an effective measure to reduce the risk of transfusion-related transmission of ZIKV (as opposed to blood-center-documented deferral which would require 
adding a question to the donor questionnaire). They also recommended the use of donor information sheets to enhance postdonation symptom reporting to blood centers to facilitate quarantine and recall of potentially infectious components from ill donors with exposure in epidemic settings throughout the tropics. ${ }^{45}$

\section{Microcephaly and Intracranial Calcifications}

Microcephaly is a head size that is smaller than expected for age. It has a variety of etiologies. It is associated with intellectual disability, developmental delay, and seizures. ${ }^{47}$ It may be evident at birth (primary microcephaly) or postnatally (secondary microcephaly). Acquired congenital microcephaly, the type associated with maternal ZIKV infection, may occur due to a variety of insults to the developing fetal brain, such as intrauterine infection. ${ }^{48}$

There has not been agreement in the scientific community about the diagnostic criteria for fetal microcephaly, particularly in the context of ZIKV exposure. Prenatally, measurement of the occipitofrontal HC by ultrasound is used as a surrogate for head size. The measurement is then compared with nomograms to determine how small it is in relation to gestational age. Two standard deviations below the mean for age has been proposed; however, other authors prefer to use $3 \mathrm{SD}$, arguing that it is more clinically meaningful because of its stronger association with mental retardation. ${ }^{49,50}$ Severe microcephaly is also characterized by a disproportion between the face and skull, sloping forehead, and small brain, with the cerebral hemispheres most affected. ${ }^{49}$ Making a diagnosis of clinically relevant microcephaly is difficult because (1) mean HC varies by age, sex, and race, (2) head measurement by itself may be difficult to interpret in the setting of incorrect dating or intrauterine growth restriction, and (3) there are varying definitions of microcephaly in the literature.

The SMFM has recommended the use of SD (as opposed to percentiles) to characterize the $\mathrm{HC}$ measurement as this was the method used in prior studies relating ultrasound findings to postnatal findings. ${ }^{51}$ SMFM recommends a detailed ultrasound examination, with particular attention to the brain, for an $\mathrm{HC}>2$ SD below the mean but reserved defining isolated microcephaly as an HC 3 SD or more below the mean for gestational age, which corresponds to the 1st percentile. The SD cutoffs that SMFM recommends are based on the nomogram by Chervenak et al. ${ }^{52}$ In a subsequent publication which evaluated the performance of various cutoffs to predict microcephaly, Chervenak et al reported that an HC larger than 2 SD below the mean excluded microcephaly, and one that is smaller than $5 \mathrm{SD}$ below the mean is almost certainly indicative of pathologic microcephaly. ${ }^{53}$ Single measurements between these SD cutoffs cannot reliably diagnose or exclude pathologic microcephaly, and serial measurements over time may be more valuable. Because the Chervenak et al nomogram does not include data for fetuses less than 20 weeks of gestation, one can use the SD cutoffs based on the nomogram by Kurmanavicius et al, if needed before 20 weeks. ${ }^{54}$ Unlike the nomogram by Chervenak et al, how- ever, the one by Kurmanavicius et al was not validated in actual cases with microcephaly and was derived from a mostly low-risk population in Switzerland. For this reason, and to be consistent with the SMFM statement, we recommend using the Chervenak et al nomogram after 20 weeks of gestation.

In comparing it to gestational age nomograms, most ultrasound reporting software characterizes the HC in percentiles rather than SD. As a practical approach, we propose the following: for fetuses at 12 weeks of gestation or greater, if the $\mathrm{HC}$ on the ultrasound reporting software is above the 5th percentile, then the fetus does not have microcephaly as the measurement will never be smaller than $2 \mathrm{SD}^{51}$ If the measurement is at or below the 5th percentile by the ultrasound reporting software, then refer to - Table 1, which lists values for SD, adapted from both the Chervenak et al and Kurmanavicius et al nomograms.

There is an alternative to the SD approach. A recent multicenter cohort study reported nomograms for various fetal biometry measurements, including HC (the NICHD National Fetal Growth Study), obtained from serial ultrasounds in more than 1,700 pregnancies. ${ }^{55}$ The study derived percentiles at all gestational ages and for various racial/ethnic categories. Given that the results of this study may be more applicable to current fetal biometry, and that it provides percentiles for all gestational ages, it may be more practical to use the 3rd percentile for race/ethnicity as a surrogate for 2 SD below the mean (-Table 2). The National Fetal Growth Study did not report cutoffs lower than the 3rd percentile; therefore, we cannot provide the percentile cutoff equivalent to $3 \mathrm{SD}$ and $5 \mathrm{SD}$. If a woman has poor obstetric dating, one option would be to assess the ratio of $\mathrm{HC}$ to abdominal circumference $(A C)$ to avoid misdiagnosing microcephaly. We have provided the 3rd percentile for $\mathrm{HC}$ to $\mathrm{AC}$ ratios by gestational age and race/ethnicity from the National Fetal Growth Study ( - Table 3).

A qualitative evaluation of the intracranial structures can be a helpful addition to biometry because morphologic derangements often accompany microcephaly, and their presence portends a worse outcome. ${ }^{49}$ Regardless of the definition, it is understood that the prognosis worsens with increasing severity of microcephaly..$^{49,52,53}$ It is important to remember that while ZIKV is currently attracting a lot of attention, there are numerous etiologies of microcephaly, including other intrauterine infections and genetic disorders, and a proper diagnostic work-up should be undertaken, including fetal karyotyping.

It is also important to note that a large percentage of fetuses with microcephaly diagnosed prenatally by ultrasound will not have pathologic microcephaly at birth. ${ }^{53}$ When the $\mathrm{HC}$ is between 2 and $3 \mathrm{SD}$ below mean for gestational age, assuming otherwise normal intracranial anatomy, follow-up ultrasound is warranted but the chance of pathologic microcephaly is low. Even using the definition of 3 SD below the mean for gestational age, only 60\% of infants will have the diagnosis at birth. ${ }^{56}$ Therefore, as the SMFM publication warns, be cautious in making the diagnosis of microcephaly prenatally, particularly when it will lead to 
Table 2 Third percentile for head circumference for each gestational age according to race/ethnicity

\begin{tabular}{|c|c|c|c|c|}
\hline \multirow[b]{2}{*}{ Gestational age (wk) } & \multicolumn{4}{|c|}{ Head circumference $(\mathrm{mm})$ 3rd percentile } \\
\hline & Non-Hispanic white & Non-Hispanic black & Hispanic & Asian/Pacific Islander \\
\hline 10 & 41.4 & 40.6 & 38.7 & 39.9 \\
\hline 11 & 52.2 & 51.7 & 49.2 & 50.6 \\
\hline 12 & 63.9 & 63.6 & 60.7 & 62.2 \\
\hline 13 & 76.1 & 75.9 & 72.7 & 74.3 \\
\hline 14 & 88.4 & 88.3 & 84.9 & 86.6 \\
\hline 15 & 100.7 & 100.5 & 97.0 & 98.9 \\
\hline 16 & 112.7 & 112.3 & 108.9 & 110.9 \\
\hline 17 & 124.4 & 123.7 & 120.4 & 122.6 \\
\hline 18 & 135.8 & 135.0 & 131.7 & 133.9 \\
\hline 19 & 147.1 & 146.3 & 142.9 & 145.1 \\
\hline 20 & 158.6 & 157.7 & 154.2 & 156.4 \\
\hline 21 & 170.2 & 169.1 & 165.6 & 167.8 \\
\hline 22 & 181.7 & 180.4 & 176.9 & 179.3 \\
\hline 23 & 193.2 & 191.5 & 188.1 & 190.7 \\
\hline 24 & 204.4 & 202.3 & 199.2 & 201.9 \\
\hline 25 & 215.4 & 212.8 & 209.9 & 212.9 \\
\hline 26 & 226.1 & 223.0 & 220.4 & 223.6 \\
\hline 27 & 236.3 & 232.8 & 230.5 & 233.8 \\
\hline 28 & 246.1 & 242.2 & 240.1 & 243.6 \\
\hline 29 & 255.3 & 251.1 & 249.3 & 252.8 \\
\hline 30 & 264.0 & 259.6 & 258.0 & 261.3 \\
\hline 31 & 272.1 & 267.4 & 266.1 & 269.2 \\
\hline 32 & 279.5 & 274.6 & 273.5 & 276.4 \\
\hline 33 & 286.2 & 280.9 & 280.2 & 282.9 \\
\hline 34 & 292.1 & 286.5 & 286.1 & 288.8 \\
\hline 35 & 297.3 & 291.1 & 291.2 & 294.0 \\
\hline 36 & 301.7 & 294.9 & 295.5 & 298.5 \\
\hline 37 & 305.4 & 298.1 & 299.0 & 302.3 \\
\hline 38 & 308.6 & 300.9 & 301.9 & 305.2 \\
\hline 39 & 311.2 & 303.5 & 304.3 & 307.3 \\
\hline 40 & 313.4 & 306.1 & 306.2 & 308.3 \\
\hline
\end{tabular}

Source: Adapted from Buck Louis et al. ${ }^{55}$

interventions that may result in adverse consequences. When there is concern for small HC, a detailed sonographic evaluation of the fetal brain should be performed, as intracranial anomalies would raise the suspicion for pathologic microcephaly and ZIKV infection.

We should also keep in mind that microcephaly may just be the most severe form of fetal involvement that could have been detected given the current ascertainment methods, and may represent just the tip of the iceberg. ${ }^{37,57}$ More subtle effects of ZIKV infection in the fetal brain may not be revealed until later stages of childhood when learning and/or visual deficits can be quantified. More systematic and longer-term follow-up is needed to determine whether there are other more subtle fetal effects, particularly in fetuses with borderline small head sizes. The implementation of IgM testing for cord or infant blood (which is not confounded by past maternal Flavivirus infections) is needed to establish fetal infections and evaluate long-term outcomes.

Finally, intracranial calcifications are a nonspecific finding. They can be associated with intrauterine infections that can cause fetal brain insults, such as toxoplasmosis and cytomegalovirus. $^{58}$ 
Table 3 Third percentile for head circumference to abdominal circumference ratio (HC/AC) for each gestational age according to race/ethnicity

\begin{tabular}{|c|c|c|c|c|}
\hline \multirow[b]{2}{*}{ Gestational age (wk) } & \multicolumn{4}{|l|}{ HC/AC 3rd percentile } \\
\hline & Non-Hispanic white & Non-Hispanic black & Hispanic & Asian/Pacific Islander \\
\hline 10 & 1.219 & 1.182 & 1.142 & 1.198 \\
\hline 11 & 1.209 & 1.183 & 1.148 & 1.192 \\
\hline 12 & 1.194 & 1.178 & 1.146 & 1.181 \\
\hline 13 & 1.177 & 1.167 & 1.138 & 1.167 \\
\hline 14 & 1.158 & 1.153 & 1.127 & 1.150 \\
\hline 15 & 1.138 & 1.137 & 1.113 & 1.132 \\
\hline 16 & 1.118 & 1.120 & 1.098 & 1.115 \\
\hline 17 & 1.099 & 1.104 & 1.083 & 1.098 \\
\hline 18 & 1.083 & 1.089 & 1.069 & 1.082 \\
\hline 19 & 1.068 & 1.077 & 1.057 & 1.068 \\
\hline 20 & 1.057 & 1.067 & 1.048 & 1.057 \\
\hline 21 & 1.047 & 1.060 & 1.041 & 1.049 \\
\hline 22 & 1.040 & 1.053 & 1.035 & 1.042 \\
\hline 23 & 1.034 & 1.048 & 1.031 & 1.037 \\
\hline 24 & 1.028 & 1.043 & 1.026 & 1.032 \\
\hline 25 & 1.023 & 1.038 & 1.022 & 1.028 \\
\hline 26 & 1.017 & 1.033 & 1.017 & 1.023 \\
\hline 27 & 1.011 & 1.026 & 1.011 & 1.017 \\
\hline 28 & 1.004 & 1.018 & 1.003 & 1.009 \\
\hline 29 & 0.995 & 1.008 & 0.993 & 1.000 \\
\hline 30 & 0.984 & 0.996 & 0.981 & 0.989 \\
\hline 31 & 0.972 & 0.982 & 0.968 & 0.976 \\
\hline 32 & 0.958 & 0.968 & 0.953 & 0.962 \\
\hline 33 & 0.944 & 0.952 & 0.937 & 0.948 \\
\hline 34 & 0.928 & 0.937 & 0.921 & 0.933 \\
\hline 35 & 0.912 & 0.921 & 0.905 & 0.919 \\
\hline 36 & 0.896 & 0.905 & 0.890 & 0.905 \\
\hline 37 & 0.880 & 0.889 & 0.874 & 0.890 \\
\hline 38 & 0.865 & 0.873 & 0.859 & 0.874 \\
\hline 39 & 0.851 & 0.857 & 0.844 & 0.857 \\
\hline 40 & 0.839 & 0.841 & 0.842 & 0.839 \\
\hline
\end{tabular}

Source: Adapted from Buck Louis et al. ${ }^{55}$

What Can Other Arboviruses Contribute to Our Understanding of Zika Virus Infection in Pregnancy?

\section{Dengue Virus}

There are some data suggesting mother-to-child (MTC) transmission of DENV, ${ }^{59-63}$ but the exact route (transplacental, breast milk, intrapartum, or close contact with newborn) has not been well established. There are data confirming the presence of infectious DENV in breast milk. ${ }^{59}$

\section{Chikungunya Virus}

CHIKV is an alphavirus not closely related to ZIKV but shares many epidemiologic properties and symptomatology with ZIKV. A review article of CHIKV in pregnancy reported evidence for transplacental transmission in the first and second trimester, leading to fetal infection and miscarriage, albeit very rarely. However, active maternal infection within 4 days of delivery conveys a high risk of MTC transmission. ${ }^{64} \mathrm{~A}$ French study observed neurocognitive outcomes at roughly 2 years of age in children of mothers with CHIKV infection in 
pregnancy. ${ }^{65}$ They also reported epidemiological associations between infection and neurodevelopmental delay rates in affected populations. Of note, there were 12 cases of chikungunya neonatal encephalitis, of which 5 developed microcephaly and 4 developed cerebral palsy. Magnetic resonance imaging studies of these children revealed white matter restrictions, particularly in the frontal lobes, a similar distribution to what Oliveira et al describe in the ZIKV patients. ${ }^{32}$ The findings with CHIKV infection during pregnancy underscore the importance of more in-depth and longer-term follow-up of the children potentially exposed to maternal infections.

\section{West Nile Virus}

West Nile virus (WNV) has been shown to be transmittable through breast milk ${ }^{66}$ and transplacentally. ${ }^{67}$ The CDC published data of 72 infants born to women with known WNV infections during pregnancy. Infants were assessed at delivery and through 12 months of age. While the CDC reported several malformations, none of the children had conclusive evidence of congenital WNV infection. ${ }^{68}$

\section{Clinical Considerations and Recommendations}

Q: How do I counsel women about travel to endemic areas? A: Pregnant women should avoid any unnecessary travel to ZIKV-endemic areas (-Fig. 1). For those who cannot avoid travel to endemic areas, great care should be taken to prevent mosquito bites.

\begin{tabular}{|ll|}
\hline Caribbean & Mexico \\
Barbados & Cape Verde \\
Curacao & \\
Gominican Republic & Puerto Rico \\
Haiti & \\
Jamaica & The Pacific Islands \\
Martinique & American Samoa \\
Saint Martin & Samoa \\
U.S. Virgin Islands & Tonga \\
& \\
Central America & South America \\
Costa Rica & Bolivia \\
El Salvador & Brazil \\
Guatemala & Colombia \\
Honduras & Ecuador \\
Nicaragua & French Guiana \\
Panama & Guyana \\
& Paraguay \\
& Suriname \\
& Venezuela \\
& \\
\hline
\end{tabular}

Fig. 1 Countries identified by the CDC as endemic to Zika virus, as of February 24, 2016. For most current information, see the CDC Web site: http://wwwnc.cdc.gov/travel/notices/.
Q: What are the signs and symptoms of ZIKV infection?

A: ZIKV infection can cause a relatively benign illness characterized by acute onset of fever, headache, malaise, arthralgia, maculopapular rash, and conjunctivitis. Albeit rare, there have been reports of Guillain-Barré syndrome following suspected ZIKV infection. ${ }^{23}$ The vast majority of ZIKV infections, however, are asymptomatic. ${ }^{14-16}$

\section{Q: Which pregnant women are at risk of infection?}

A: Anyone living in or traveling to the regions in which there is transmission by the vector is at risk. - Fig. 1 lists the endemic areas identified by the $\mathrm{CDC}$ at the time of submitting this article. The most up-to-date list is found on the CDC Web site (http://wwwnc.cdc.gov/travel/notices/). However, there is a lag in reporting due to the time required to complete diagnostics and report results. Cases of sexual transmission have been reported. ${ }^{27,28}$ While sexual transmission is likely much rarer than mosquito vector transmission, pregnant women engaging in sexual intercourse with men living in or traveling to endemic areas are at some degree of risk.

\section{Q: What risks does infection pose to the fetus?}

A: Current data are limited to case reports and personal communications which describe microcephaly, white matter atrophy, intracranial calcifications, and ocular findings in fetuses with varying degrees of evidence of intrauterine infection. However, we do not know the incidence of ZIKV infection in pregnant women, the transmission rate to the fetus, or the rate of brain insult or other sequelae of fetal infection.

Q: How do we prevent infection and is there a treatment? A: Until vaccines become available, prevention of mosquito bites in endemic areas is the principal form of prevention. For those who cannot avoid travel to endemic areas, great care should be taken to prevent mosquito bites. Mosquito netting should be used indoors. When outside, wear long sleeves and pants, wear permethrin-treated clothing, and use an Environmental Protection Agency-registered insect repellant, such as $\mathrm{N}, \mathrm{N}$-diethyl-meta-toluamide (DEET) or picaridin, which are safe to use during pregnancy and lactation. ${ }^{69}$ The benefits of protection from mosquitos in endemic areas outweigh any hypothetical risks of permethrin and DEET to a developing fetus. Eliminate areas of standing water near the home. With the reports of sexual transmission and presence of ZIKV in semen, the CDC recommends pregnant women practice abstinence to eliminate the risk or use condoms correctly and consistently to minimize the risk of sexual transmission. ${ }^{9}$ The $\mathrm{CDC}$ goes on to advise women of reproductive age to take the same precautions if they live in an endemic area or if their sexual partner lives in or has traveled to an endemic area. ${ }^{9}$ There is no antiviral treatment for ZIKV infection. Supportive care involves rest, hydration, analgesics, and antipyretics, such as acetaminophen. Avoid nonsteroidal anti-inflammatory drugs (NSAIDS) even in non-pregnant patients.

Q: What testing is available and how do we order them? A: According to the CDC, laboratory evidence of maternal ZIKV infection can include viral RNA detected by RT-PCR in 


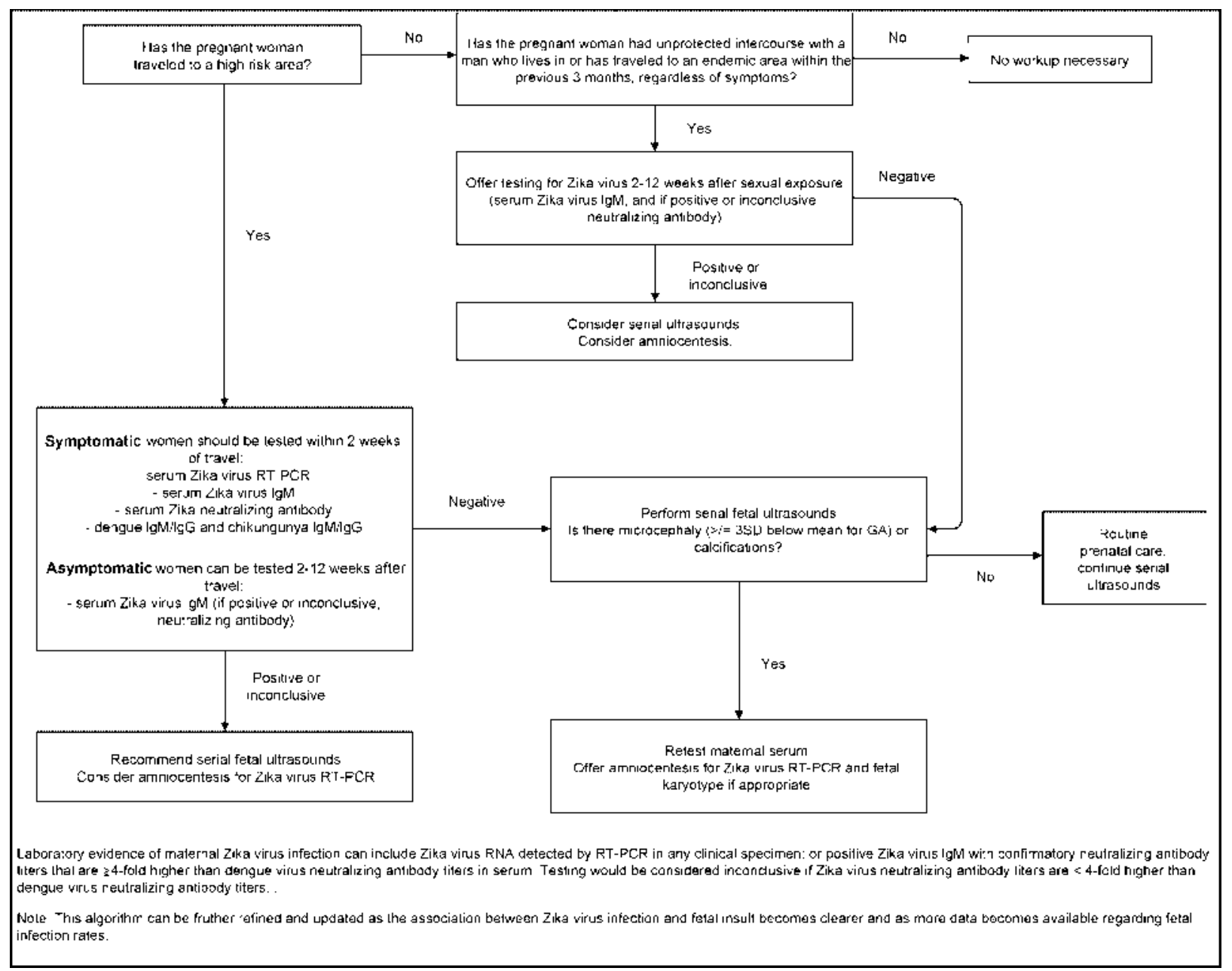

Fig. 2 Management of pregnant women who are at risk of exposure to ZIKV due to travel or sexual contact.

any clinical specimen (within 7 days of symptom onset for serum) or positive serum IgM tests with confirmatory neutralizing antibody titers that are fourfold or more higher than DENV neutralizing antibody titers in serum ( $\geq 4$ days after symptom onset). Testing would be considered inconclusive if ZIKV neutralizing antibody titers are less than fourfold than DENV neutralizing antibody titers. ${ }^{6}$ The only complete testing available in the United States for ZIKV is performed at the CDC Arbovirus Diagnostic Laboratory and a few state health departments. Please see the CDC Diagnostic Testing webpage for specific information about specimen collection and delivery (http://www.cdc.gov/zika/hc-providers/diagnostic.html). In brief, the $\mathrm{CDC}$ requests the following:

- Clinical information: date of onset of symptoms, date of specimen collection, pertinent travel history, and the patient's name

- Acute specimen, if possible (3-10 days after onset of symptoms)

- Convalescent specimen, if possible (2-3 weeks after acute sample)

We recommend calling your local or state health department immediately to determine what samples are needed and how they should be stored and shipped, prior to encountering a patient, as their requirements may differ from the $\mathrm{CDC}$ and they may be overwhelmed with high volume.

\section{Q: How do I manage a woman I have identified as at risk due to travel in an endemic area?}

A: Please see $\boldsymbol{- F i g . ~} 2$ for our proposed management algorithm which was adapted from the CDC guidance for women traveling in endemic areas. ${ }^{70}$ Women who have traveled to an endemic area during or within 2 weeks of pregnancy and who report symptoms consistent with ZIKV infection should be tested for viral RNA with serum RT-PCR (within 7 days of symptoms onset) and should be tested for exposure to the virus with a ZIKV IgM assay (4 or more days after symptom onset). Positive IgM testing should be confirmed with neutralizing antibody detection and also warrants testing to evaluate for DENV and CHIKV (dengue IgG and IgM, chikungunya $\operatorname{IgG}$ and $\operatorname{IgM}$ ) as there is extensive cross-reactivity among their antibodies in serologic testing, and CHIKV is endemic in the same regions where ZIKV is circulating. The interim CDC guidance released on February 5, 2016 states that pregnant women residing in non-DENV-endemic regions who have traveled to a ZIKV-endemic area but do not report 
symptoms consistent with ZIKV infection can be tested for Zika IgM 2 to 12 weeks after travel, and if positive, confirmation via detection of neutralizing antibody. ${ }^{70}$ However, prior exposure to DENV or other Flaviviruses, or yellow fever vaccination, will likely confound interpretation of serologic results. This underscores the need to take a thorough history from the patient regarding travel and vaccine history.

While the CDC states that women who traveled in an endemic area and remained asymptomatic "can" be offered blood testing, one has to keep in mind that interpretation of these laboratory test results is complex. Practices may decide whether to offer testing for these women or not based on the a priori risk of infection. If the testing is offered, patients should be made aware that false-positives and false-negatives are possible. In patients where the a priori risk of infection is very low, such as asymptomatic women who traveled to affected areas, most of the positive tests are likely to be false-positive. A negative serum IgM test does not exclude infection as it may take several weeks after maternal infection to become positive. Before ordering serologic testing, patients should be counseled regarding the interpretation of the results and the potential for false-positives and false-negatives. Until more data are available, we recommend continuing serial ultrasound even if serologic testing is negative, particularly if done less than 12 weeks after potential exposure. If blood testing is positive or inconclusive, we recommend serial fetal ultrasounds and advise consideration of amniocentesis for ZIKV RT-PCR testing.

If the patient has not had an ultrasound yet in the pregnancy, we recommend a detailed ultrasound: while microcephaly and calcifications have been reported thus far, we do not know whether there may be other findings associated with ZIKV infection that have not yet been reported. If she has had a prior ultrasound during the current pregnancy, simple follow-up growth ultrasounds with surveillance for microcephaly and intracranial calcifications will suffice. If either microcephaly or intracranial calcifications are identified, the mother should be retested and offered amniocentesis for ZIKV RT-PCR and fetal genetic testing, including microarray in the case of microcephaly.

\section{Q: How do I manage a woman who is residing in an endemic area and reports symptoms consistent with ZIKV infection?}

A: The $\mathrm{CDC}$ has published guidelines for pregnant women who are residing in an endemic area. ${ }^{70}$ While the CDC algorithm recommends routine prenatal care if serologic testing and the first ultrasound are normal, we believe serial fetal ultrasounds in the setting of negative serologic testing in this subset of women is warranted given difficulty interpreting serologic test results, as we describe above. Therefore, pregnant women who reside in an endemic area and report symptoms consistent with ZIKV infection should be tested for ZIKV with serum RT-PCR (within 7 days of symptom onset), ZIKV IgM, and neutralizing antibody (4 or more days after symptom onset), as well as testing to evaluate for DENV and CHIKV (dengue IgG and IgM, chikungunya IgG and IgM) as there is cross-reactivity among their antibodies in serologic testing. If the testing is positive or inconclusive, we recommend serial fetal ultrasounds and advise consideration of amniocentesis for ZIKV testing. If serum testing is negative, we recommend serial fetal ultrasound. If ultrasound reveals either microcephaly or intracranial calcifications, the maternal serum should be retested and amniocentesis offered. If serial ultrasounds do not identify abnormalities, retest maternal serum in the midsecond trimester.

Q: How do I manage a woman who is residing in an endemic area and does not report symptoms consistent with ZIKV infection?

A: The CDC has published guidelines for pregnant women who are residing in an endemic area. ${ }^{69}$ Our recommendations differ in that we believe serial ultrasounds are warranted given the limitations and difficulty in interpreting serologic testing, as well as the high percentage of asymptomatic infections with unknown fetal transmission rate. Therefore, pregnant women who reside in an endemic area but do not report symptoms consistent with ZIKV infection can be tested at their first prenatal visit for serum ZIKV IgM, and if IgM is positive or indeterminate, for neutralizing antibodies. Serologic testing in this population may be difficult to interpret as women may have had previous exposures to other arboviruses, as noted above. The local health department that is performing the testing will assist you in interpreting the results. If the testing is positive or inconclusive, we recommend performing serial ultrasounds and consideration of amniocentesis for ZIKV testing. If the blood testing is negative, we recommend serial ultrasounds. If ultrasound reveals either microcephaly or intracranial calcifications, the maternal serum should be retested and amniocentesis offered. If serial ultrasounds do not identify abnormalities, retest maternal serum in the midsecond trimester.

\section{Q: How should I manage pregnant women at risk of sexual transmission of ZIKV?}

A: The evidence of sexual transmission is limited to three cases. $^{27,28}$ Very little is understood about the incidence and duration of viral shedding in the male genitourinary tract. Given this information and the lack of knowledge about the natural history of MTC transmission, pregnant women should be counseled to avoid intercourse (vaginal, anal, and oral) and/or use barrier protection with partners living or traveling in the endemic areas for the remainder of pregnancy. ${ }^{9}$ If your pregnant patient has already engaged in unprotected intercourse with a man who lives in or has traveled to an endemic area within the previous 3 months, her risk of infection is unknown, though likely very low. She may be managed similar to a woman who traveled to an endemic area, particularly if her sexual contact was symptomatic or had confirmation of ZIKV infection.

\section{Q: What sonographic fetal findings associated with ZIKV infection are we looking for?}

A: The fetus should be evaluated for $\mathrm{HC}$ and intracranial calcifications. Microcephaly is defined as an HC 3 SD below the mean for gestational age, or smaller, for pregnancies at 
12 weeks or beyond ( - Table 1 ). ${ }^{51-53}$ A qualitative evaluation of the intracranial structures can be a helpful addition to biometry because morphologic derangements often accompany microcephaly. It is important to remember that while ZIKV is attracting a lot of attention, there are numerous etiologies of microcephaly, and a proper diagnostic work-up should be undertaken, including fetal karyotyping. Outside of the ZIKV literature, microcephaly is usually not diagnosed until the midsecond or third trimester. ${ }^{71} \mathrm{HC}$ between 2 and 3 SD below the mean, or between 3rd and 5th percentile should have a follow-up ultrasound in 3 to 4 weeks to determine the trajectory.

\section{Q: Who should be offered an amniocentesis?}

A: Pregnant women who have been exposed to ZIKV, by either residence in or travel to an endemic area or sexual contact with a man who is at risk of ZIKV infection, who have positive blood testing or concerning fetal ultrasound findings should be offered amniocentesis after 15 weeks of gestation. You should counsel the patient that (1) there is a $0.5 \%$ risk of fetal loss associated with amniocentesis, (2) when considering infant and child outcome, the predictive value of a positive or negative RT-PCR on amniotic fluid is still unknown, and (3) there is no antiviral treatment for ZIKV infection. See - Fig. 2 for management algorithm.

Q: If maternal and/or fetal Zika infection is diagnosed or suspected, is there anything I should do after delivery?

A: The CDC recommends specific testing for (1) infants with microcephaly or intracranial calcifications born to women who traveled to or resided in an area with ZIKV transmission while pregnant, and (2) infants born to mothers with positive or inconclusive test results for ZIKV infection. ${ }^{72}$ The majority of this testing will be ordered by the pediatrician or neonatologist. However, the obstetrician should send the placenta and umbilical cord (or fetal remains in case of a fetal loss) for histopathologic evaluation, with ZIKV immunohistochemical staining on fixed tissue and ZIKV RT-PCR of fixed and frozen tissue and cord serum. This testing is performed at the CDC and the Web site provides detailed instructions on the preparation and sampling of these tissues (http://www.cdc.gov/zika/hcproviders/tissue-collection-submission.html).

\section{Q: Is long-term follow-up necessary?}

A: Postpartum follow-up is not required for the mother. There is no evidence to suggest that prior ZIKV infection puts a women at risk for birth defects in future pregnancies, as viremia is only thought to last for 7 days. ${ }^{21}$ Long-term follow-up for the infant is recommended by the $\mathrm{CDC}$. It is crucial that the obstetrician notify the pediatrician or neonatologist of any potential ZIKV exposure the infant may have had in utero, as immediate testing of the newborn is recommended, as above. ${ }^{72}$

\section{Conclusion}

ZIKV is a mosquito-borne pathogen, currently causing an epidemic in Central and South America. Infection is usually asymptomatic but can cause a relatively benign, self-limited illness in an adult, with rare case reports of suspected neurologic sequelae, such as Guillain-Barré syndrome. ${ }^{23}$ There is no antiviral treatment and no vaccine. There are extremely limited data about ZIKV in pregnancy and fetal/ neonatal effects. Case reports support intrauterine transmission and an association between maternal ZIKV infection and fetal microcephaly and calcifications. Epidemiological investigations are ongoing in Brazil and elsewhere to further examine this link. While there is no conclusive evidence, there are some data to suggest that vertical transmission of ZIKV via breast milk is possible, as well. ${ }^{26}$ While there is no evidence that the sequelae of neonatal ZIKV infection are any more severe than the benign adult illness, and the CDC argues that the benefits of breastfeeding outweigh the risk of transmission, ${ }^{72}$ there are no published data on short- or long-term outcomes of neonatal or infant infection. Therefore, studies on the infectiousness of breast milk and sequelae of neonatal infection are urgently needed. Cases of sexual transmission have been reported, ${ }^{27,28}$ and therefore, until the duration of ZIKV persistence in semen is known, abstinence or barrier protection is recommended for pregnant women for the entirety of the pregnancy ${ }^{9}$ and for men at risk of infection who are having intercourse with women of reproductive capacity. Women of reproductive age who are at risk of infection should be aware of the risks of infection in pregnancy and, therefore, should take measures to prevent pregnancy in the near future. Measures to protect the blood supply from asymptomatic viremic donations are warranted. Prevention of mosquito bites, however, is the principal preventative approach, as the most likely mode of transmission is by the vector. Review of other related viruses in pregnancy may improve our understanding of possible modes of ZIKV transmission and sequelae of maternal, fetal, and neonatal infection.

\section{Areas of Future Research}

When the Emergency Committee of the International Health Regulations advised the Director-General of the WHO to declare the cluster of microcephaly and other neurological disorders a Public Health Emergency of International Concern (PHEIC), ${ }^{11}$ they did so based on the fact that so little is known about these clusters that are associated in time and place with ZIKV outbreaks. ${ }^{73}$ With so many unanswered questions about ZIKV and its effects on pregnancy and fetal development, there is a need for rapid and collaborative research in all areas: basic science, diagnostics, epidemiology, clinical medicine, and public health. Specific aims of future research are not limited to but would include (1) better ascertainment of cases and outcomes with long-term follow-up of exposed mothers and infants (including cohort and case-control studies with collection of appropriate potential confounder data), (2) development of better diagnostic tools given severe limitations of RT-PCR and serologic testing, as well as better understanding of current tools (sensitivity, specificity, positive and negative predictive values), (3) understanding other potential modes of transmission, especially breastfeeding and sexual contact, (4) understanding the role of asymptomatic 
infection (risk of transmission, especially in pregnancy), (5) animal models to definitely prove or disprove causal link between maternal ZIKV infection and fetal brain insult, (6) novel methods of vector control, and (7) vaccine and antiviral pharmaceutical development.

\section{Conflict of Interest}

None.

\section{References}

1 Agencia Saude. Microcefalia: Ministerio da Saude divulga boletim epidemiologico. 2015. Available at: http://portalsaude.saude.gov. br/index.php/cidadao/principal/agencia-saude/20805-ministerioda-saude-divulga-boletim-epidemiologico. Accessed January 23, 2016

2 Pan American Health Organization. Epidemiological Alert: Neurological Syndrome, Congenital Malformations, and Zika Virus Infection. Implications for Public Health in the Americas. Washington, DC: World Health Organization, Pan American Health Organization; December 1, 2015. Available at: http://www.paho. org/hq/index.php?option=com_docman\&task=doc_download\&Itemid=\&gid=32405\&lang=en. Accessed January 29, 2016

3 European Centre for Disease Prevention and Control. Rapid Risk Assessment: Microcephaly in Brazil Potentially Linked to the Zika Virus Epidemic - 24 November 2015. Stockholm: ECDC; 2015

4 European Centre for Disease Prevention and Control. Rapid Risk Assessment: Zika Virus Epidemic in the Americas: Potential Association with Microcephaly and Guillain-Barré Syndrome 10 December 2015. Stockholm: ECDC; 2015

5 European Centre for Disease Prevention and Control. Rapid Risk Assessment. Zika Virus Disease Epidemic: Potential Association with Microcephaly and Guillain-Barré Syndrome. Second update, 8 February 2016. Stockholm: ECDC; 2016

6 Centers for Disease Control and Prevention, Division of VectorBorne Diseases, Arboviral Diseases and Dengue Branches. Updated diagnostic testing for Zika, chikungunya, and dengue viruses in US Public Health Laboratories (Memorandum). January 13, 2016. Available at: http://www.aphl.org/Materials/CDCMemo_Zika_Chik_Deng_Testing_011916.pdf. Assessed January 20, 2016

7 Petersen EE, Staples JE, Meaney-Delman D, et al. Interim guidelines for pregnant women during a Zika virus outbreak United States, 2016. MMWR Morb Mortal Wkly Rep 2016; 65(2):30-33

8 Society of Maternal Fetal Medicine. Practice advisory: updated interim guidance for care of obstetric patients during a Zika virus outbreak. February 12, 2016. Available at: https://www.smfm. org/publications/220-acog-smfm-joint-practice-advisory-interim-guidance-for-care-of-obstetric-patients-during-a-zika-virusoutbreak. Accessed February 12, 2016.

9 Oster AM, Brooks JT, Stryker JE, et al. Interim guidelines for prevention of sexual transmission of Zika virus - United States, 2016. MMWR Morb Mortal Wkly Rep 2016;65(5):120-121

10 Pan American Health Organization. Preliminary Guidelines for the Surveillance of Microcephalia in Newborns in Settings with Risk of Circulation of the Zika Virus. Washington, DC: World Health Organization, Pan American Health Organization; January 21, 2016. Available at: http://www.paho.org/hq/index.php?option=com_content\&view=article\&id=11620\%3Avirus-zika-and-possiblelink-to-microcefalia\&catid=8424\%3Acontent\&Itemid=41711\&lan$\mathrm{g}=\mathrm{en}$. Accessed February 10, 2016

11 WHO. WHO statement of the first meeting of the International Health Regulations (2005) (IHR 2005) Emergency Committee on Zika virus and observed increase in neurological disorders and neonatal malformations. February 1, 2016. http://www.who.int/ mediacentre/news/statements/2016/1st-emergency-committeezika/en/. Accessed February 2, 2016.

12 CDC. CDC Emergency Operations Center moves to highest level of activation for Zika response. February 3, 2016. Available at: http:// www.cdc.gov/media/releases/2016/s0208-zika-eoca-activation. html. Accessed February 10, 2016

13 Kuhn JH, Peters CJ. Arthropod-borne and Rodent-borne virus infections. In: Kasper D, Fauci A, Hauser S, Longo D, Jameson J, Loscalzo J eds. Harrison's Principles of Internal Medicine. 19th ed. New York, NY: McGraw-Hill; 2015

14 Ioos S, Mallet HP, Leparc Goffart I, Gauthier V, Cardoso T, Herida M. Current Zika virus epidemiology and recent epidemics. Med Mal Infect 2014;44(7):302-307

15 Hayes EB. Zika virus outside Africa. Emerg Infect Dis 2009;15(9): $1347-1350$

16 Duffy MR, Chen TH, Hancock WT, et al. Zika virus outbreak on Yap Island, Federated States of Micronesia. N Engl J Med 2009;360(24): 2536-2543

17 Dick GW. Zika virus. II. Pathogenicity and physical properties. Trans R Soc Trop Med Hyg 1952;46(5):521-534

18 Cao-Lormeau VM, Roche C, Teissier A, et al. Zika virus, French polynesia, South pacific, 2013. Emerg Infect Dis 2014;20(6): 1085-1086

19 Kuno G, Chang GJ. Full-length sequencing and genomic characterization of Bagaza, Kedougou, and Zika viruses. Arch Virol 2007; 152(4):687-696

20 Faye O, Freire CC, Iamarino A, et al. Molecular evolution of Zika virus during its emergence in the 20(th) century. PLoS Negl Trop Dis 2014;8(1):e2636

21 Lanciotti RS, Kosoy OL, Laven JJ, et al. Genetic and serologic properties of Zika virus associated with an epidemic, Yap State, Micronesia, 2007. Emerg Infect Dis 2008;14(8):1232-1239

22 Enfissi A, Codrington J, Roosblad J, Kazanji M, Rousset D. Zika virus genome from the Americas. Lancet 2016;387(10015):227-228

23 Oehler E, Watrin L, Larre P, et al. Zika virus infection complicated by Guillain-Barre syndrome-case report, French Polynesia, December 2013. Euro Surveill 2014;19(9):2072

24 Musso D, Nhan T, Robin E, et al. Potential for Zika virus transmission through blood transfusion demonstrated during an outbreak in French Polynesia, November 2013 to February 2014. Euro Surveill 2014;19(14):20761

25 Marano G, Pupella S, Vaglio S, Liumbruno GM, Grazzini G. Zika virus and the never-ending story of emerging pathogen and transfusion medicine. Blood Transfus 2015:1-6

26 Besnard M, Lastère S, Teissier A, Cao-Lormeau V, Musso D. Evidence of perinatal transmission of Zika virus, French Polynesia, December 2013 and February 2014. Euro Surveill 2014;19(13): 20751

27 Foy BD, Kobylinski KC, Chilson Foy JL, et al. Probable non-vectorborne transmission of Zika virus, Colorado, USA. Emerg Infect Dis 2011;17(5):880-882

28 Musso D, Roche C, Robin E, Nhan T, Teissier A, Cao-Lormeau VM. Potential sexual transmission of Zika virus. Emerg Infect Dis 2015; 21(2):359-361

29 Musso D, Roche C, Nhan TX, Robin E, Teissier A, Cao-Lormeau VM. Detection of Zika virus in saliva. J Clin Virol 2015;68:53-55

30 Prince HE, Matud JL. Estimation of dengue virus IgM persistence using regression analysis. Clin Vaccine Immunol 2011;18(12): 2183-2185

31 CDC. Surveillance and control of Aedes aegypti and Aedes albopictus in the United States. Available at: http://www.cdc.gov/chikungunya/resources/vector-control.html. Accessed on February 8, 2016

32 Oliveira Melo AS, Malinger G, Ximenes R, Szejnfeld PO, Alves Sampaio S, Bispo de Filippis AM. Zika virus intrauterine infection causes fetal brain abnormality and microcephaly: tip of the iceberg? Ultrasound Obstet Gynecol 2016;47(1):6-7 
33 Calvet G, Aguiar RS, Melo ASO, et al. Detection and sequencing of Zika virus from amniotic fluid of fetuses with microcephaly in Brazil: a case study. Lancet Infect Dis 2016 (e-pub ahead of print). doi:10.1016/S1473-3099(16)00095-5

34 Bell TM, Field EJ, Narang HK. Zika virus infection of the central nervous system of mice. Arch Gesamte Virusforsch 1971;35(2): 183-193

35 Tetro JA. Zika and microcephaly: causation, correlation, or coincidence? Microbes Infect 2016 (e-pub ahead of print). doi:10.1016/j. micinf.2015.12.010

36 Ventura CV, Maia M, Bravo-Filho V, Góis AL, Belfort R Jr. Zika virus in Brazil and macular atrophy in a child with microcephaly. Lancet 2016;387(10015):228

37 de Paula Freitas B, de Oliveira Dias JR, Prazeres J, et al. Ocular findings in infants with microcephaly associated with presumed Zika virus congenital infection in Salvador, Brazil.JAMA Ophthalmol 2016(e-pub ahead of print). doi:10.1001/jamaophthalmol. 2016.0267

38 Mlakar J, Korva M, Tul N, et al. Zika virus associated with microcephaly. N Engl J Med 2016 (e-pub ahead of print)

39 Martines RB, Bhatnagar J, Keating MK, et al. Notes from the field: evidence of Zika virus infection in brain and placental tissues from two congenitally infected newborns and two fetal losses - Brazil, 2015. MMWR Morb Mortal Wkly Rep 2016;65(6):159-160

40 Pan American Health Organization, World Health Organization. Question and Answers: Zika and pregnancy. Available at: http:// www.paho.org/Hq/index.php?option=com_content\&view=article\&id=11552\%3Aquestion-and-answers-zika-and-pregnancy\&catid $=8424 \% 3$ Acontent $\&$ Itemid $=41711$ \&lang $=$ en. Accessed January 22, 2016

41 Schuler-Faccini L, Ribeiro EM, Feitosa IM, et al; Brazilian Medical Genetics Society-Zika Embryopathy Task Force. Possible association between Zika virus infection and microcephaly - Brazil, 2015. MMWR Morb Mortal Wkly Rep 2016;65(3):59-62

42 Dontigny L, Arsenault MY, Martel MJ, et al; Society of Obstetricians and Gyneacologist of Canada. Rubella in pregnancy. J Obstet Gynaecol Can 2008;30(2):152-168

43 Lipitz S, Yinon Y, Malinger G, et al. Risk of cytomegalovirusassociated sequelae in relation to time of infection and findings on prenatal imaging. Ultrasound Obstet Gynecol 2013;41(5): 508-514

44 Meaney-Delman D, Hills SL, Williams C, et al. Zika Virus Infection Among U.S. Pregnant Travelers-August 2015-February 2016. Morb Mortal Wkly Rep 2016(epub ahead of print). doi: http:// dx.doi.org/10.15585/mmwr.mm6508e1er

45 AABB. Association Bulletin \#16-03. Zika, dengue, and chikungunya viruses. Available at: https://www.aabb.org/programs/publications/bulletins/Documents/ab16-03.pdf. Accessed February 23, 2016

46 Spenser BR, Stramer SL, Dodd RY, et al. Survey to estimate donor loss to 14- or 28-day travel deferral for mitigation of CHIKV, DENV and other acute infections. Presented at: AABB Annual Meeting; October 24-27, 2015; Anaheim, CA

47 Ashwal S, Michelson D, Plawner L, Dobyns WB; Quality Standards Subcommittee of the American Academy of Neurology and the Practice Committee of the Child Neurology Society. Practice parameter: evaluation of the child with microcephaly (and evidence based review): report of the Quality Standards Subcommittee of the American Academy of Neurology and the Practice Committee of the Child Neurology Society. Neurology 2009; 73(11):887-897

48 Passemard S, Kaindl AM, Verloes A. Microcephaly. In: Dulac O, Lassonde M, Sarnat HB eds. Handbook of Clinical Neurology, Vol III, Pediatric Neurology Part I. Amsterdam, the Netherlands: Elsevier; 2013:129-141

49 Pilu G. Ultrasound evaluation of the fetal neural axis. In: Callen PW, ed. Ultrasound in Obstetrics and Gynecology. 5th ed. Philadelphia, PA: Saunders; 2008:382-383
50 Microcephaly. In: Bianchi DW, Crombleholme TM, D’Alton ME, Malone FD, eds. Fetology: Diagnosis and Management of the Fetal Patient. 2nd ed. New York, NY: McGraw Hill; 2010:166-171

51 SMFM. SMFM statement: ultrasound screening for fetal microcephaly following Zika virus exposure. Am J Obstet Gynecol 2016 (e-pub ahead of print). doi:10.1016/j.ajog.2016.02.043. Available at: http://www.ajog.org/pb/assets/raw/Health\%20Advance/journals/ymob/SMFM\%20Statement_Fetal\%20microcephaly.pdf. Accessed February 18, 2016

52 Chervenak FA, Jeanty P, Cantraine F, et al. The diagnosis of fetal microcephaly. Am J Obstet Gynecol 1984;149(5):512-517

53 Chervenak FA, Rosenberg J, Brightman RC, Chitkara U, Jeanty P. A prospective study of the accuracy of ultrasound in predicting fetal microcephaly. Obstet Gynecol 1987;69(6):908-910

54 Kurmanavicius J, Wright EM, Royston P, et al. Fetal ultrasound biometry: 1. Head reference values. Br J Obstet Gynaecol 1999; 106(2):126-135

55 Buck Louis GM, Grewal J, Albert PS, et al. Racial/ethnic standards for fetal growth: the NICHD Fetal Growth Studies. Am J Obstet Gynecol 2015;213(4):449.e1-449.e41

56 Leibovitz Z, Daniel-Spiegel E, Malinger G, et al. Microcephaly at birth - the accuracy of three references for fetal head circumference. How can we improve prediction? Ultrasound Obstet Gynecol 2015 (e-pub ahead of print). doi:10.1002/uog.15801

57 Jampol LM, Goldstein DA. Zika virus infection and the eye. JAMA Ophthalmol 2016 (e-pub ahead of print). doi:10.1001/ jamaophthalmol.2016.0284

58 Leite JM, Granese R, Jeanty P, Herbst SS. Fetal syndromes. In: Callen PW ed. Ultrasound in Obstetrics and Gynecology. 5th ed. Philadelphia, PA: Saunders; 2008:133-135

59 Barthel A, Gourinat AC, Cazorla C, Joubert C, Dupont-Rouzeyrol M, Descloux E. Breast milk as a possible route of vertical transmission of dengue virus? Clin Infect Dis 2013;57(3):415-417

60 Chen LH, Wilson ME. Transmission of dengue virus without a mosquito vector: nosocomial mucocutaneous transmission and other routes of transmission. Clin Infect Dis 2004;39(6): e56-e60

61 Sirinavin S, Nuntnarumit P, Supapannachart S, Boonkasidecha S, Techasaensiri C, Yoksarn S. Vertical dengue infection: case reports and review. Pediatr Infect Dis J 2004;23(11): 1042-1047

62 Basurko C, Carles G, Youssef M, Guindi WE. Maternal and fetal consequences of dengue fever during pregnancy. Eur J Obstet Gynecol Reprod Biol 2009;147(1):29-32

63 Tan PC, Rajasingam G, Devi S, Omar SZ. Dengue infection in pregnancy: prevalence, vertical transmission, and pregnancy outcome. Obstet Gynecol 2008;111(5):1111-1117

64 Dotters-Katz SK, Grace MR, Strauss RA, Chescheir N, Kuller JA. Chikungunya fever: obstetric considerations on an emerging virus. Obstet Gynecol Surv 2015;70(7):453-457

65 Gérardin P, Sampériz S, Ramful D, et al. Neurocognitive outcome of children exposed to perinatal mother-to-child Chikungunya virus infection: the CHIMERE cohort study on Reunion Island. PLoS Negl Trop Dis 2014;8(7):e2996

66 Centers for Disease Control and Prevention (CDC). Possible West Nile virus transmission to an infant through breast-feedingMichigan, 2002. MMWR Morb Mortal Wkly Rep 2002;51(39): 877-878

67 Nguyen Q Morrow C, Novick L, et al; Centers for Disease Control and Prevention (CDC). Intrauterine West Nile virus infection-New York, 2002. MMWR Morb Mortal Wkly Rep 2002;51(50): 1135-1136

68 O'Leary DR, Kuhn S, Kniss KL, et al. Birth outcomes following West Nile Virus infection of pregnant women in the United States: 2003-2004. Pediatrics 2006;117(3):e537-e545

69 Nasci RS, Wirtz RA, Brogdon WG. Protection against mosquitos, ricks, and other arthropods. In: CDC Health Information for International Travel, 2016. New York, NY: Oxford University Press; 
2015:94-99. Available at: http://wwwnc.cdc.gov/travel/yellowbook/2016/the-pre-travel-consultation/protection-against-mosquitos-ticks-other-arthropods

70 Oduyebo T, Petersen EE, Rasmussen SA, et al. Update: interim guidelines for health care providers caring for pregnant women and women of reproductive age with possible Zika virus exposure - United States, 2016. MMWR Morb Mortal Wkly Rep 2016;65(5): $122-127$
71 Bromley B, Benacerraf BR. Difficulties in the prenatal diagnosis of microcephaly. J Ultrasound Med 1995;14(4):303-306

72 Staples JE, Dziuban EJ, Fischer M, et al. Interim guidelines for the evaluation and testing of infants with possible congenital Zika virus infection - United States, 2016. MMWR Morb Mortal Wkly Rep 2016;65(3):63-67

73 Heymann DL, Hodgson A, Sall AA, et al.Zika virus and microcephaly: why is this situation a PHEIC? Lancet 2016;387(10020):719-721 